\title{
Infección por el HTLV-I en pacientes con síndromes linfoproliferativos en dos sitios centinela de Cuba
}

\author{
Héctor M. Díaz Torres, ${ }^{1}$ Nereyda Álvarez Vega, ${ }^{2}$ \\ Jorge E. Muñío Perurena, ${ }^{3}$ Ana Luisa Lubián Caballero, ${ }^{1}$ \\ Dayamí Martín Alfonso, ${ }^{1}$ Dervel F. Díaz Herrera ${ }^{1}$ \\ y Madelín Blanco de Armas ${ }^{1}$
}

Forma de citar

Díaz Torres HM, Álvarez Vega N, Muñío Perurena JE, Lubián Caballero AL, Martín Alfonso D, Díaz Herrera DF, et al. Infección por el HTLV-I en pacientes con síndromes linfoproliferativos en dos sitios centinela de Cuba. Rev Panam Salud Publica. 2010;27(1):17-22.

RESUMEN Objetivo. Determinar la frecuencia de la infección por el HTLV-I en pacientes con síndromes linfoproliferativos, así como en sus familiares y contactos sexuales, en dos sitios de vigilancia centinela en Cuba.

Métodos. Se analizaron todos los pacientes que tenían un diagnóstico presuntivo de neoplasias hematológicas entre enero de 1996 y enero de 2007 atendidos en los servicios de hematología del Hospital Hermanos Ameijeiras (HHA), de Ciudad de La Habana, y el Hospital Provincial Comandante Faustino Pérez (HPCFP), de Matanzas, Cuba. Se determinó la seropositividad al HTLV-I por ELISA y western blot y se confirmó la infección mediante la reacción en cadena de la polimerasa. Se estudiaron también los familiares y los contactos sexuales de los pacientes positivos. Se utilizó la prueba de la Z para la comparación de proporciones.

Resultados. La seroprevalencia de la infección por el HTLV-I en pacientes con sindromes linfoproliferativos fue de 0,4\%, mayor en el HPCFP que en el HHA $(6,1 \%$ frente a 0,2\%; $\mathrm{P}<0,001)$. No se encontraron diferencias significativas en la frecuencia de la infección según la edad, el sexo y el color de la piel. De los 53 familiares y contactos sexuales estudiados, 8 $(15,1 \%)$ tuvieron diagnóstico positivo de infección por el HTLV-I.

Conclusión. La frecuencia de la infección por el HTLV-I en el grupo estudiado fue superior a la encontrada con anterioridad en Cuba. Se confirmó la utilidad de la vigilancia seroepidemiológica mediante centros centinela.

Palabras clave Virus linfotrópico de células T humanas tipo 1; anticuerpos anti-HTLV-I; trastornos linfoproliferativos; vigilancia de guardia; estudios seroepidemiológicos; Cuba.

1 Laboratorio de Investigaciones del Sida, San José de las Lajas, La Habana, Cuba. La correspondencia se debe dirigir a Héctor Manuel Díaz Torres, Laboratorio de Investigaciones del Sida, Carretera a Tapaste y Autopista Nacional, San José de Las Lajas, La Habana, Cuba. Correo electrónico: cicdc@infomed.sld.cu

2 Hospital Provincial Comandante Faustino Pérez, Matanzas, Cuba.

3 Hospital Clínico Quirúrgico Hermanos Ameijeiras, Ciudad de La Habana, Cuba.
El virus linfotrópico de las células $\mathrm{T}$ humanas tipo I (HTLV-I) fue el primer retrovirus humano identificado y el primero asociado con una neoplasia maligna. Su aislamiento se realizó en 1978 en los Estados Unidos de América a partir de un paciente con linfoma cutáneo de células $\mathrm{T}$, aunque no se informó hasta 1980 (1). Posteriormente, el mismo pa- ciente desarrolló una leucemia/linfoma de células T del adulto (LLTA), que en 1977 investigadores japoneses habían descrito como una nueva enfermedad de probable etiología viral asociada con una susceptibilidad genética específica individual $(2,3)$. Luego se estableció que los virus aislados de pacientes estadounidenses y japoneses eran idénticos y que 
correspondían al HTLV-I, aceptado como agente causal de la LLTA (4).

Suficientes datos apoyan el papel etiológico del HTLV-I en la LLTA: la infección por este virus precede al desarrollo de la enfermedad, se puede identificar mediante técnicas serológicas y ensayos de ácidos nucleicos y el HTLV-I transforma los linfocitos CD4+ en cultivo, da lugar a un fenotipo celular con expresión genética similar a la que se observa en los linfocitos de enfermos con LLTA y se integra al genoma de los linfocitos CD4+ (5). Sin embargo, la gama de enfermedades etiológicamente relacionadas con este retrovirus parece ser más amplia y se reconoce su participación en la patogenia de la uveítis, el síndrome de Sjögren, la polimiositis y la sinovitis, así como en algunas enfermedades autoinmunitarias, como la paraparesia espástica tropical (PET) y la mielopatía asociada con el HTLV-I (6).

La infección por el HTLV-I es endémica en la cuenca del Caribe, América Central, América del Sur, África, Asia y la Melanesia. Se transmite por la infusión de sangre y derivados de la sangre contaminados, mediante el contacto sexual y perinatalmente de madre a hijo; en este último caso, la lactancia materna por tiempo prolongado se considera el factor más importante (7). Aunque la mielopatía asociada con la infección por el HTLV-I puede adquirirse por cualquiera de estas vías de transmisión, la LLTA suele presentarse varias décadas después de haber recibido lactancia de una madre infectada (8).

En las regiones endémicas, la LLTA y la PET constituyen las principales enfermedades indicadoras de la circulación del virus en la población. En el Caribe, varios estudios poblacionales han detectado tasas de seroprevalencia de $3 \%$ a $6 \%$ de infección por el HTLV-I; la PET se presenta con mayor frecuencia que la LLTA (9).

En Cuba, en estudios seroepidemiológicos realizados entre 1989 y 1990 en pacientes con hemopatías malignas no se detectaron casos infectados por el HTLV-I (10, 11). Sin embargo, en 1990 -en el marco de una investigación que formaba parte de los primeros esfuerzos para establecer un sistema de vigilancia basado en sitios centinela de atención clínica- se diagnosticó por primera vez esta infección en una paciente de la provincia de Matanzas que presentaba la forma aguda de LLTA. Este caso se in- formó años más tarde (12), pero el diagnóstico de la enfermedad motivó la inmediata continuación de estudios seroepidemiológicos. Entre 1991 y 1996 se estudiaron 26352 personas con diferentes conductas o factores de riesgo, de las cuales 10 presentaban la infección por el HTLV-I, para una seroprevalencia en esta población de 0,037\% (13). Aunque ese valor es mucho menor que el informado en países vecinos (9), este estudio demostró que el HTLV-I estaba presente en la población cubana y confirmó la necesidad de continuar atentos a su posible diseminación.

Como parte de la vigilancia epidemiológica de la infección por el HTLV-I en la población cubana, se establecieron sitios centinela para realizar el tamizaje sistemático de los pacientes con enfermedades probablemente relacionadas con esa infección. El objetivo del presente trabajo fue determinar la frecuencia de la infección por el HTLV-I en pacientes con síndromes linfoproliferativos, así como en sus familiares y contactos sexuales, en dos sitios de vigilancia centinela en Cuba.

\section{MATERIALES Y MÉTODOS}

En 1996 se estableció la vigilancia centinela de la infección por el HTLV-I en los servicios de hematología del Hospital Hermanos Ameijeiras (HHA), de Ciudad de La Habana, y el Hospital Provincial Comandante Faustino Pérez (HPCFP), de Matanzas, Cuba. Posteriormente se amplió la vigilancia al estudio serológico de los familiares y los contactos sexuales de los pacientes con alguna de las enfermedades hematológicas asociadas con la infección por el HTLV-I que resultaron positivos para esa infección durante el tamizaje de vigilancia.

\section{Selección de las personas a estudiar}

Se analizaron todos los pacientes que tenían un diagnóstico presuntivo de neoplasia hematológica en ambos sitios de vigilancia entre enero de 1996 y enero de 2007. En el HHA se incluyeron los pacientes con síndromes linfoproliferativos de diversos tipos, independientemente de la provincia de residencia, y se excluyeron los enfermos con diagnóstico de hemopatías no malignas. En el HPCFP se incluyeron los pacientes residentes en la provincia de Matanzas con diagnóstico de síndrome linfoproliferativo crónico caracterizado por leucocitosis, linfocitosis y presencia de linfocitos atípicos, y se excluyeron los pacientes con diagnóstico de linfoma de Hodgkin, linfoma de células B y leucemia sin marcadores de proliferación de linfocitos $\mathrm{T}$.

Una vez obtenido el consentimiento informado de los pacientes, los familiares y los contactos relacionados, se tomaron $4 \mathrm{~mL}$ de sangre periférica de cada uno y se les aplicó una encuesta epidemiológica, según el Protocolo de Trabajo para la Infección y la Enfermedad por Retrovirus Humanos aprobado por la Comisión de Aplicación del Manual de Prácticas Médicas del Hospital Hermanos Ameijeiras (14). Este protocolo establece las acciones médicas a seguir con los pacientes y las personas seropositivas asintomáticas, entre ellas la obtención del consentimiento informado y la forma de realizar las consejerías anterior y posterior a la prueba de diagnóstico. Los familiares se agruparon para el análisis en: madres (no se localizaron padres), hijos, hermanos y otros familiares (tíos y primos).

Los enfermos con neoplasias hematológicas recibieron atención médica especializada en los servicios de hematología de los hospitales centinela. Las personas seropositivas asintomáticas recibieron atención continua en las consultas externas de esos hospitales y se les ofrecieron servicios de consejería, acciones de promoción de salud y vigilancia clínica, hematológica y neurológica, según los protocolos de trabajo del HHA (14) y el Programa Nacional de Control de las Infecciones de Transmisión Sexual, el VIH y el sida (15).

\section{Estudio serológico}

El suero de las personas estudiadas se conservó a $-20{ }^{\circ} \mathrm{C}$ hasta su envío al Laboratorio de Investigaciones del Sida -que funciona como Laboratorio Nacional de Referencia para el Diagnóstico de Retrovirus-, ubicado en San José de las Lajas, La Habana, para realizar las pruebas diagnósticas.

La detección de anticuerpos contra el HTLV-I se realizó mediante los sistemas de ELISA y western blot DAVIH HTLV-I y DAVIH BLOT HTLV-I, respectivamente, según las indicaciones del fabricante (Laboratorios DAVIH, La Habana, Cuba). Los resultados se interpretaron según las recomendaciones de la Organización Mundial de la Salud: las muestras se consideraron positivas cuando resultaban 
reactivas en dos ensayos independientes por ELISA y presentaban reactividad combinada en el western blot contra productos de los genes env y gag; se estableció el diagnóstico positivo de infección por el HTLV-I cuando dos muestras independientes de la misma persona, tomadas en días diferentes, resultaron repetidamente positivas según el criterio anterior. Para confirmar el diagnóstico, a los pacientes se les tomó una muestra de $10 \mathrm{~mL}$ de sangre total con EDTA para detectar el ADN viral mediante la reacción en cadena de la polimerasa (PCR) con cebadores específicos.

\section{Detección de ADN viral}

El ADN de las personas a estudiar se obtuvo de células mononucleares de sangre periférica por el método de la proteinasa $\mathrm{K}$ con SDS; se extrajo con fenol, cloroformo y alcohol isoamílico; y se precipitó en etanol. Se emplearon tres pares diferentes de oligonucleótidos cebadores específicos para el HTLV-I: 1) HTL 116/117 (5' TCT ATC CTC CAA GGC CTG GA 3' y 5' TGA CAA GCC CGC AAC ATA TC $3^{\prime}$ ) para el gen gag; 2) HTL 140/141 (5' CGG ATA CCC AGT CTA CGT GT 3' y 5' GAG CCG ATA ACG CGT CCA TCG 3') que amplifican una región del gen tax; y 3) HTL 128/129 (5' CTT CAC AGT CTC TAC TGT GC 3' y 5' CGG CAG TTC TGT GAC AGG G $\left.3^{\prime}\right)$ que amplifican una región del gen pol $(2,4)$. La PCR de cada muestra se realizó por duplicado en $10 \mu \mathrm{L}$ de tampón Taq 10x, $200 \mu \mathrm{M}$ de cada dNTP (Promega, Madison, Estados Unidos), 100 pmol de cada cebador, $1 \mu \mathrm{g}$ del ADN extraído de la muestra y 2,5 unidades de polimerasa Taq (Promega), en un volumen final de $50 \mu \mathrm{L}$ (12). Como control positivo se empleó ADN de células MT2 infectadas crónicamente con el HTLV-I y como control negativo, células mononucleares de sangre periférica de donantes de sangre no infectados.

Se empleó un termociclador modelo PTC-100-60 (MJ Research, Waltham, MA, Estados Unidos) con un programa para amplificar el gen gag (30 ciclos de 3 min de desnaturalización a $95^{\circ} \mathrm{C}, 1 \mathrm{~min}$ de anillamiento a $45^{\circ} \mathrm{C}$ y $1 \mathrm{~min}$ de extensión a $72{ }^{\circ} \mathrm{C}$, con un paso final de extensión de $10 \mathrm{~min}$ a $72{ }^{\circ} \mathrm{C}$ ) y otro programa para la amplificación de los genes tax y pol (30 ciclos de 1 min de desnaturalización a $95^{\circ} \mathrm{C}, 1 \mathrm{~min}$ de anillamiento a $55^{\circ} \mathrm{C}$ y 1 min de extensión a $72{ }^{\circ} \mathrm{C}$, con un paso final de extensión de $10 \mathrm{~min}$ a $\left.72{ }^{\circ} \mathrm{C}\right)$.

Los productos de la reacción se guardaron a $4{ }^{\circ} \mathrm{C}$ y se visualizaron mediante electroforesis en un gel de agarosa tipo VII al 3\% teñido con $0,5 \mu \mathrm{g} / \mathrm{mL}$ de bromuro de etidio. Como patrón de pesos moleculares se aplicaron $50 \mu \mathrm{g}$ del plásmido pGEM $^{\circledR}$ digerido con Hint I, Rsa I y $\operatorname{Sln}$ I (Promega), que produce bandas desde 2645 pares de bases hasta 36 pares de base.

Se hizo un seguimiento mediante PCR de los casos con resultados indeterminados en las pruebas serológicas.

\section{Procesamiento de datos}

Los datos recogidos en la encuesta epidemiológica y la historia clínica de cada paciente, así como los resultados de laboratorio, se introdujeron en una base de datos elaborada en Microsoft Office Excel 2003. Se utilizó la prueba de la Z para la comparación de proporciones. Los datos se procesaron con el programa para análisis epidemiológico de datos tabulados Epidat versión 3.1 (16).

\section{RESULTADOS}

En el cuadro 1 se presentan los resultados del diagnóstico de la infección por el HTLV-I en pacientes con enfermedades hematológicas atendidos en los dos sitios centinela. En el HHA se detectaron dos pacientes, el primero con diagnóstico confirmado de LLTA y el segundo, admitido por una posible neoplasia del sistema hematopoyético, recibió finalmente el diagnóstico de amiloidosis sistémica primaria. En el HPCFP se diagnosticaron tres enfermos, todos con LLTA. La frecuencia resultó mayor en el HPCFP $(6,1 \%)$ que en el HHA $(0,2 \%$;
$P<0,001)$; no se encontraron diferencias significativas en la frecuencia de la infección por el HTLV-I según la edad, el sexo y el color de la piel en ambos sitios de vigilancia. Durante el seguimiento, no se observó seroconversión de ninguno de los casos con resultados indeterminados en las pruebas serológicas.

La mediana de la edad de los cinco pacientes, todos hombres, fue de 56,0 años, mientras que la de los cuatro enfermos con LLTA fue de 57,5 años. Predominaron las personas con color de la piel negra y de ascendencia africana. Los cuatro pacientes con LLTA presentaron la forma aguda de la enfermedad y recibieron poliquimioterapia específica para el linfoma de células $\mathrm{T}$ desde el momento del diagnóstico hematológico. A pesar del tratamiento, la sobrevida promedio desde la fecha del diagnóstico clínico de LLTA fue de solo 2 meses (cuadro 2). El paciente con amiloidosis primaria se incluyó en el estudio con el diagnóstico presuntivo de síndrome linfoproliferativo por presentar adenopatías y aumento de células plasmáticas en la médula ósea. Posteriormente, este paciente desarrolló una miocardiopatía restrictiva con manifestaciones renales y articulares; se descartaron las discrasias de células plasmáticas y las posibles causas de amiloidosis sistémica reactiva. El diagnóstico definitivo fue amiloidosis sistémica primaria, con resultado positivo a la prueba del rojo congo y presencia de proteína monoclonal de cadena ligera.

No se pudo completar el tamizaje serológico de todos los familiares y contactos (cuadro 3). Del paciente 1, el padre y la madre, procedentes de las islas Guadalupe y Haití, respectivamente, habían fallecido por enfermedades aparentemente no relacionadas con la infección

CUADRO 1. Resultados de la vigilancia epidemiológica de la infección por el HTLV-I en dos centros centinela, Cuba, enero de 1966 a enero de 2007

\begin{tabular}{cccc}
\hline Sitio de vigilancia & $\begin{array}{c}\text { Personas } \\
\text { estudiadas }\end{array}$ & $\begin{array}{c}\text { Pacientes con } \\
\text { infección por } \\
\text { el HTLV-I }\end{array}$ & $\begin{array}{c}\text { Seropositividad } \\
(\%)^{\mathrm{a}}\end{array}$ \\
\hline HHA $^{\mathrm{b}}$ & 1232 & 2 & 0,2 \\
HPCFPc $_{\text {Total }}$ & 49 & 3 & $6,1^{\mathrm{c}}$ \\
& 1281 & 5 & 0,4 \\
\hline
\end{tabular}

a Diferencia significativa entre ambos hospitales, según la prueba de la Z para la comparación de proporciones $(P=0,001$; intervalo de confianza de $95 \%$ : 0,13 a 0,90$)$.

b HHA: Hospital Clínico Quirúrgico Hermanos Ameijeiras, Ciudad de La Habana. Criterio de inclusión: todos los síndromes linfoproliferativos.

c HPCFP: Hospital Provincial Comandante Faustino Pérez, Matanzas. Criterio de inclusión: síndrome linfoproliferativo crónico con leucocitosis, linfocitosis y presencia de linfocitos atípicos. 
CUADRO 2. Enfermedades asociadas y variables de interés clínico-epidemiológico en los pacientes con infección por el HTLV-I, Cuba, enero de 1966 a enero de 2007

\begin{tabular}{|c|c|c|c|c|c|}
\hline \multirow[b]{2}{*}{ Variable } & \multicolumn{5}{|c|}{ Pacientes } \\
\hline & 1 & 2 & 3 & 4 & 5 \\
\hline Diagnósticoa & LLTA aguda & LLTA aguda & $\begin{array}{l}\text { Amiloidosis } \\
\text { sistémica } \\
\text { primaria }\end{array}$ & LLTA aguda & LLTA aguda \\
\hline Fecha de diagnóstico & 01/02/1996 & 17/11/1997 & $06 / 12 / 2000$ & 01/09/2003 & 17/04/2006 \\
\hline Centro centinelab & HHA & HPCFP & HHA & HPCFP & HPCFP \\
\hline Provincia de procedencia & Holguín & Matanzas & Granma & Matanzas & Matanzas \\
\hline Edad (años) & 60 & 59 & 52 & 32 & 56 \\
\hline Sexo & Hombre & Hombre & Hombre & Hombre & Hombre \\
\hline Color de la piel & Negra & Mestiza & Blanca & Negra & Negra \\
\hline Ascendencia étnica & $\begin{array}{c}\text { Caribe } \\
\text { (Guadalupe } \\
\text { y Haití) }\end{array}$ & África & $\begin{array}{c}\text { No } \\
\text { precisada }\end{array}$ & $\begin{array}{c}\text { África } \\
\text { (mandinga) }\end{array}$ & África \\
\hline Sobrevida (meses) & 4 & 2 & 6 & 1 & 1 \\
\hline
\end{tabular}

a LLTA: leucemia-linfoma de células T del adulto.

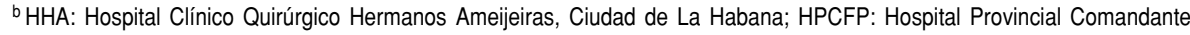
Faustino Pérez, Matanzas.

por el HTLV-I, y la esposa se negó a colaborar. Los padres del paciente 2 y los de su esposa positiva, también habían fallecido. No se localizaron todos los hermanos, los hijos y el padre del paciente 3 .

El paciente 4 no tuvo hijos y no se pudo estudiar ninguno de sus contactos sexuales, mientras que del paciente 5 no se localizaron las madres de seis de sus hijos. Los padres de estos pacientes habían fallecido.

De los 53 familiares y contactos sexuales estudiados, 8 tuvieron diagnóstico positivo de infección por el HTLV-I $(15,1 \%)$. Una de las personas diagnosticadas, prima por línea materna del paciente 4, había desarrollado PET y sinovitis proliferativa de rodilla asociadas con la infección por el HTLV-I; no había sido diagnosticada previamente.

Los ocho familiares y contactos positivos procedían de la provincia de Matanzas. Los subgrupos con mayor frecuencia de infección fueron el de los contactos sexuales y el de las madres; no se detectaron seropositivos entre los trece hijos estudiados de los casos primarios.

La frecuencia general encontrada en el estudio de familiares y contactos sexuales fue mayor que la observada en los sitios de vigilancia clínica $(15,1 \%$ frente a $0,4 \%$, respectivamente; $P<0,001)$.

\section{DISCUSIÓN}

En los primeros estudios de tamizaje de la infección por el HTLV-I en pacientes cubanos con neoplasias hematológicas no se detectaron casos positivos, por lo que se llegó a afirmar que el HTLV-I tiene una circulación muy limitada en Cuba y que, a pesar de su ubicación geográfica, no es una zona endémica de infección por el HTLV-I (9-11). Sin embargo, a diferencia de informes anteriores, en los sitios de vigilancia centinela estudiados se encontró una frecuencia de seropositividad general de $0,4 \%$, superior en el HPCFP $(6,1 \%)$ que en el HHA $(0,2 \% ; P<0,001)$.
La mayor frecuencia encontrada en el HPCFP pudiera estar relacionada con la exclusión del estudio serológico de los síndromes linfoproliferativos menos asociados con el HTLV-I y con la búsqueda activa de la infección en las personas que presentaban características clínicas de LLTA, confirmadas mediante estudio serológico cuando se comprobó que en los frotis de sangre periférica había linfocitos atípicos con alteraciones morfológicas nucleares, característicos de los casos de LLTA. Otra posible explicación es que como en Matanzas se detectó la primera paciente con LLTA diagnosticada en Cuba (12), el virus puede haber estado circulando más tiempo.

En el HHA se estudiaron personas de todas las provincias del país y se tamizaron todos los pacientes ingresados con diagnóstico presuntivo de algún tipo de síndrome linfoproliferativo, por lo que podía abarcar a personas con enfermedades no asociadas etiológicamente con el HTLV-I. No obstante, este enfoque permitió detectar la infección por el HTLV-I en el paciente 3 de esta serie, con diagnóstico de amiloidosis sistémica primaria. Este caso se hubiera pasado por alto si se hubieran seguido los criterios de inclusión y exclusión aplicados en el HPCFP. La asociación de este tipo de amiloidosis en un paciente con infección por el HTLV-I es un hallazgo de interés, ya que la amiloidosis asociada con el HTLV-I se presenta generalmente en la forma sistémica reactiva en el curso de otras enfermedades concomitantes $(17,18)$.

Los cuatro pacientes con LLTA desarrollaron la forma aguda de la enfermedad. Se han descrito cinco estadíos clínicos de la LLTA: aguda, portador asintomático, estadío preleucémico, forma latente o crónica y linfoma (19). La forma aguda se caracteriza por presentar leucocitosis, linfocitosis, elevada proporción de linfocitos

CUADRO 3. Infección por el HTLV-I en los familiares y los contactos sexuales estudiados de los pacientes detectados durante la vigilancia epidemiológica, Cuba, enero de 1966 a enero de 2007

\begin{tabular}{|c|c|c|c|c|c|c|c|c|c|c|c|c|c|c|c|}
\hline \multirow[b]{2}{*}{ Paciente } & \multicolumn{3}{|c|}{ Contactos sexuales } & \multicolumn{3}{|c|}{ Madres } & \multicolumn{3}{|c|}{ Hijos } & \multicolumn{3}{|c|}{ Hermanos } & \multicolumn{3}{|c|}{ Tíos y primos } \\
\hline & No. & Positivos & $\%$ & No. & Positivos & $\%$ & No. & Positivos & $\%$ & No. & Positivos & $\%$ & No. & Positivos & $\%$ \\
\hline 1 & 0 & $N A^{a}$ & NA & 0 & NA & NA & 3 & 0 & 0 & 7 & 0 & 0 & 0 & NA & NA \\
\hline 2 & 2 & 1 & 50,0 & 0 & NA & NA & 2 & 0 & 0 & 0 & NA & NA & 1 & 0 & 0 \\
\hline 3 & 1 & 0 & 0 & 1 & 0 & 0 & 0 & NA & NA & 1 & 0 & 0 & 0 & NA & NA \\
\hline 4 & 0 & NA & NA & 1 & 1 & 100 & 0 & NA & NA & 4 & 2 & 50,0 & 14 & 3 & 21,4 \\
\hline 5 & 2 & 1 & 50,0 & 0 & NA & NA & 8 & 0 & 0 & 0 & NA & NA & 6 & 0 & 0 \\
\hline TOTAL & 5 & 2 & 40,0 & 2 & 1 & 50,0 & 13 & 0 & 0 & 12 & 2 & 16,7 & 21 & 3 & 14,3 \\
\hline
\end{tabular}

\footnotetext{
a NA: no aplica.
} 
atípicos en la sangre periférica, características histológicas propias del linfoma no Hodgkin agresivo, osteolisis, hipercalcemia, lesiones cutáneas y corta sobrevida a pesar de la quimioterapia convencional (6). En las poblaciones donde circula el HTLV-I, la LLTA en estadio agudo se diagnostica con mayor frecuencia que las restantes formas $(20,21)$.

El promedio de edad de los pacientes con LLTA (51,8 años) se corresponde con lo informado por otros autores, posiblemente debido al largo período de incubación de esta infección. Como ya se ha dicho, se ha documentado que $10 \%$ de los infantes infectados durante la lactancia materna desarrollan la enfermedad varias décadas después (8).

La seropositividad materna solo se demostró en el más joven de los cuatro pacientes con LLTA de esta serie. El fallecimiento de las madres de los tres restantes impidió completar el estudio; sin embargo, la ascendencia caribeña de uno de ellos y africana de los demás pudieran considerarse elementos epidemiológicos que apoyarían la hipótesis de la infección vertical, ya que sus progenitores proceden de poblaciones con una elevada prevalencia de la infección por el HTLV-I (9). Por el contrario, la ausencia de esta infección en la madre del paciente con amiloidosis, descartó la infección perinatal en este paciente $\mathrm{y}$ - junto con la seronegatividad de la esposa- indicó la posibilidad de una infección menos antigua; en este caso no se pudo esclarecer la fuente de infección y no se encontraron pistas útiles para plantear una hipótesis etiológica entre la infección por el HTLV-I y la amiloidosis sistémica del paciente. Otro elemento epidemiológico de interés fue la ascendencia étnica del paciente, probablemente no africana. En este caso se podría considerar la posibilidad de una asociación casual.

Estudios moleculares han esclarecido el origen ancestral africano de las cepas del HTLV-I que circulan en América Latina (22). El color de la piel y la ascendencia étnica de los pacientes con LLTA estudiados aquí indican que el HTLV-I llegó a Cuba con personas de origen africano, ya hayan venido directamente de África o con oleadas posteriores de inmigrantes caribeños (22-24).

La LLTA aguda es una enfermedad de curso fatal y a pesar de los tratamientos más avanzados, los pacientes solo viven menos de un año después del diagnós- tico $(9,21,25)$. Sin embargo, la corta sobrevida de los participantes en este estudio puede estar también relacionada con el diagnóstico tardío de la neoplasia hematológica, determinada por la admisión hospitalaria en estadios avanzados de la enfermedad.

La alta proporción de mujeres seropositivas en el grupo de contactos sexuales $(40,0 \%)$ puede deberse al prolongado tiempo de contacto, por lo que se suman dos variables epidemiológicas conocidas: el tiempo de exposición y la mayor eficiencia de la transmisión del hombre a la mujer (9).

La ausencia de seropositivos en los 13 hijos de los casos primarios puede deberse a que no todos nacieron de madres positivas. Sin embargo, en el subgrupo de tíos y primos se detectó una alta frecuencia de seropositividad (14,3\%), posiblemente porque estos familiares estaban relacionados con los casos primarios por la línea materna, con una mayor probabilidad de haber nacido de madres infectadas. Aunque no todos los niños nacidos de madres infectadas adquieren la enfermedad (la tasa de transmisión vertical informada es de $18 \%$ a $30 \%$ ), la lactancia materna prolongada puede aumentar la probabilidad de infección (9, 26). En este estudio se confirmó la seropositividad de una tía y una prima maternas de un caso primario, lo que puede llevar a suponer que la infección ocurrió por la vía perinatal o la lactancia materna. Además, se comprobó que la prima seropositiva al HTLV-I presentaba una afección neurológica y articular, cuya etiología se pudo esclarecer en el curso de esta investigación (27).

Varios miembros de la familia del paciente 4 resultaron positivos al HTLV-I y presentaron enfermedades relacionadas con esa infección: la LLTA del caso primario y los procesos inflamatorios (PET y sinovitis) diagnosticados a su prima. En este grupo de estudio, el espectro de enfermedades asociadas con el HTLV-I abarcó algunas con diferentes mecanismos patogénicos, probablemente como respuesta a las características inmunitarias específicas de cada paciente. Esta interpretación se apoya en hallazgos de otros autores que han estudiado las manifestaciones clínicas asociadas con el HTLV-I en poblaciones con diferentes respuestas inmunitarias (28).

La búsqueda activa de la infección por el HTLV-I permitió encontrar más casos seropositivos en los familiares de los casos primarios de Matanzas que en los del HHA, donde los pacientes detectados notificaron menos tíos y primos y algunos no se pudieron localizar. Esto pudiera considerarse, no obstante, un indicador de la circulación del HTLVI en esta población.

Tanto la seropositividad observada en los sitios de vigilancia centinela como la encontrada en los estudios seroepidemiológicos con los familiares y los contactos sexuales resultaron más elevadas que las encontradas en estudios anteriores $(10,11,13)$. Aunque la frecuencia de casos positivos sea menor que las tasas de seroprevalencia en la mayoría de los países de las Américas, en Cuba también se requieren políticas y estrategias para enfrentar los desafíos que impone la circulación del HTLV-I (29).

$\mathrm{Al}$ analizar los resultados de la presente investigación se deben tener en cuenta dos limitaciones. En primer lugar, no se logró estudiar a todos los progenitores de los pacientes con LLTA y el estudio seroepidemiológico de los familiares y los contactos sexuales no fue exhaustivo. Por otra parte, el diseño de la investigación no tomó en cuenta a otros grupos que presentan factores, condiciones o conductas de riesgo, lo que impidió hacer comparaciones y estimar la prevalencia en la población.

Se concluye que la frecuencia de la infección por el HTLV-I en el grupo estudiado fue superior a la encontrada con anterioridad en Cuba. Se confirmó la utilidad de la vigilancia seroepidemiológica mediante centros centinela, ya que permitió confirmar la presencia del HTLV-I en pacientes con enfermedades asociadas con este virus; el estudio de las personas relacionadas con los casos primarios permitió llegar a un diagnóstico temprano, contribuyó a mejorar la atención médica de esa paciente y aportó una valiosa información para la intervención epidemiológica.

Se requieren estudios más amplios para establecer la magnitud real de la circulación del HTLV-I en Cuba. Se recomienda ampliar la vigilancia centinela de la infección por este retrovirus a todos los centros de atención hematológica, estudiar de forma sistemática a las personas relacionadas con los casos positivos y establecer el tamizaje de la infección por el HTLV-I en los grupos con conductas o factores de riesgo. 


\section{REFERENCIAS}

1. Poiesz BJ, Ruscetti FW, Gazdar AP, Bunn PA, Minna JD, Gallo RC. Detection and isolation of type $\mathrm{C}$ retrovirus particles from fresh and cultured lymphocytes of a patient with cutaneous T-cell lymphoma. Proc Natl Acad Asc USA. 1980;77(12):7415-9.

2. Takatsuki K, Uchiyama T, Sagawa K, Yodoi J. Adult $\mathrm{T}$ cell leukemia in Japan. In: Seno S, Takaku F, Irino S, ed. Topics in hematology. Amsterdam: Excerpta Medica; 1977. Pp. 73-7.

3. Uchiyama T, Yodoi J, Sagawa K, Takatsuki K, Uchino H. Adult T cell leukemia: clinical and hematologic features of 16 cases. Blood. 1977;50:481-92.

4. Yoshida M. Discovery of HTLV-1, the first human retrovirus, its unique regulatory mechanisms, and insights into pathogenesis. Oncogene. 2005;24:5931-7.

5. Franchini G, Nicot C, Johnson JM. Seizing of $\mathrm{T}$ cells by human T-cell leukemia/lymphoma virus type I. Adv Cancer Res. 2003;89:69-132.

6. Ohshima K. Pathological features of diseases associated with human T-cell leukemia virus type I. Cancer Sci. 2007;98(6):772-8.

7. Hino S, Katamine S, Miyata H, Tsuji Y, Yamabe T, Miyamoto T, et al. Primary prevention of HTLV-I in Japan. J Acquir Immune Defic Syndr Hum Retrovirol. 1996;13(1):S199-203.

8. Ratner L. Human T cell lymphotropic virus associated leukemia/lymphoma. Curr Opin Oncol. 2005;17:469-73.

9. Manns A, Hisada M, La Grenade L. Human T-lymphotropic virus type I infection. Lancet. 1999;353:1951-8.

10. Navea L, Silva E, Rivero R, Hernández P. Pesquisaje de anticuerpos contra el virus HTLV-1 en hemopatías malignas. Rev Cubana Hematol Inmunol Hemoter. 1990;6(1):101-6.

11. Hernández P, Rivero R, Ballester M, Navea L, Matutes E, Catovsky D, et al. Very low seroprevalence of HTLV-I/II in Cuba: antibodies in blood donors and in hematological and non-hematological patients. Vox Sang. 1991; 61:277-8.

12. Muñío JE, Díaz HM, Carnot J, De Castro R, Navea L, Rodríguez I. Leucemia/linfoma T del adulto. Primer caso en Cuba. Rev Cubana Med. 2003;42(3).

13. Lubián AL, Díaz HM, Silva E, Pérez MT, Cruz $\mathrm{O}$, de la Fuente JL, et al. Seroprevalencia de la infección por HTLV-I en diferentes grupos de riesgo estudiados en Cuba. Rev Cubana Med. 1998;37(4):199-204.

14. Díaz HM. Infección y enfermedad por retrovirus humanos. Manual de Prácticas Médicas del Hospital Hermanos Ameijeiras. 2. ${ }^{a}$ ed. Ciudad de La Habana: Hospital Hermanos Ameijeiras; 2008. Hallado en http://www. hospitalameijeiras.sld.cu/hha/mpm/. Acceso el 26 de noviembre de 2009.

15. República de Cuba, Ministerio de Salud Pública. Programa Nacional de Prevención y Control de las ITS-VIH-sida. Informe nacional sobre los progresos realizados en la aplicación del UNGASS. Ciudad de La Habana: MINSAP; 2008.

16. Xunta de Galicia, Organización Panamericana de la Salud. Epidat 3.1: análisis epidemiológico de datos tabulados. Santiago de Compostela: Xunta de Galicia, OPS; 2006. Hallado en http:/ / dxsp.sergas.es/ApliEdatos/Epidat/cas/ default.asp. Acceso el 26 de noviembre de 2009.

17. Seguchi T, Kyoraku Y, Saita K, Ihi T, Nagai M, Akiyama $Y$, et al. Human T-cell lymphotropic virus type I (HTLV-I) associated myelopathy and Sjögren's syndrome representing pulmonary nodular amyloidosis and multiple bullae: report of an autopsy case. Virchows Arch. 2006;448(6):874-6.

18. Takeshita K, Yamada S, Sato N, Kuwabara K, Asano K, Yamaguchi K. An unusual case of mediastinal lymphadenopathy caused by amyloidosis. Intern Med. 2000;39(10):839-42.

19. Kibler KV, Jean KT. Human T-cell leukemia virus type I: 25 years of progress and challanges. J Biomed Sci. 2005;12:7-11.

20. Proietti FA, Carneiro-Proietti AB, CatalanSoarez BC, Murphy EL. Global epidemiology of HTLV-I infection and associated diseases. Oncogene. 2005;24:6058-68.

21. Shuh M, Beilke M. The human T-cell leukemia virus type 1 (HTLV-1): new insights into the clinical aspects and molecular pathogenesis of adult T-cell leukemia/lymphoma (ATLL) and tropical spastic paraparesis/ HTLV-associated myelophaty (TSP/HAM). Microsc Res Tech. 2005;176-96.

22. Mota AC, Van Dooren $S$, Fernandes FM Pereira SA, Queiroz AT, Gallazzi VO, et al The close relationship between South African and Latin American HTLV type 1 strains corroborated in a molecular epidemiological study of the HTLV type 1 isolates from a blood donor cohort. AIDS Res Hum Retroviruses. 2007;23 (4):503-7.

23. Franco JL. Comercio clandestino de esclavos. Ciudad de La Habana: Ciencias Sociales; 1996.

24. Gómez R, Chailloux G, Whitney R, Chang F, Corrales M. De dónde son los cubanos. Ciudad de La Habana: Ciencias Sociales; 2007.

25. Hermine O, Wattel E, Gessain A, Bazarbachi A. Adult $\mathrm{T}$ cell leukemia, a review of established and new treatments. BioDrugs. 1998; 10:447-62.

26. Gessain A, Mahieux R. A virus called HTLVI. Epidemiological aspects. Presse Med. 2000; 29(40):2233-9.

27. Díaz HM, Sánchez J, Martín D, Pérez J. Paraparesia espástica tropical y sinovitis proliferativa asociadas al HTLV-I. Rev Cubana Med Trop. 2008;60(3). Hallado en http://bvs.sld. $\mathrm{cu} /$ revistas/mtr/vol60_3_08/mtr14308.htm. Acceso el 26 de noviembre de 2009.

28. Goedert JJ, Li HC, Gao XJ, Chatterjee N, Sonoda S, Biggar RJ, et al. Risk of human T-lymphotropic virus type I-associated diseases in Jamaica with common HLA types. Int J Cancer. 2007;121(5):1092-7.

29. Carneiro-Proietti AB, Catalan-Soares BC, Castro-Costa CM, Murphy EL, Sabino EC. HTLV in the Americas: challenges and perspectives. Rev Panam Salud Publica. 2006; 19(1):44-53.

Manuscrito recibido el 12 de junio de 2008. Aceptado para publicación, tras revisión, el 9 de diciembre de 2008.
ABSTRACT

\section{Human T-cell lymphotropic virus type I infection in patients with \\ lymphoproliferative disorders at two sentinel sites in Cuba}

Objective. To determine the prevalence of human T-cell lymphotropic virus type I (HTLV-I) infection among patients with lymphoproliferative disorders, as well as among their family members and sexual contacts, at two sentinel sites in Cuba.

Methods. An analysis was conducted of all the patients with a presumptive diagnosis of hematological malignancies seen by the hematology departments of the Hospital Hermanos Ameijeiras (HHA), City of Havana, and the Hospital Provincial Comandante Faustino Pérez (HPCFP), Matanza, Cuba, in January 1996-January 1997. HTLV-I seropositivity was determined by ELISA and Western Blot, and infection was confirmed by polymerase chain reaction. The positive patients' family members and sexual contacts were also assessed. The Z-test was used to compare proportions.

Results. Seroprevalence of HTLV-I infection in patients with lymphoproliferative disorders was $0.4 \%$ higher at the HPCFP than at the HHA $(6.1 \%$ versus $0.2 \%$, $P<0.001)$. There were no significant differences in prevalence by age, sex, or skin color. Of the 53 family members and sexual contacts studied, 8 (15.1\%) were positive for HTLV-I infection.

Conclusion. The prevalence of HTLV-I in the study group was higher than previously found in Cuba. The value of seroepidemiological surveillance through sentinel sites was confirmed.

Key words Human T-lymphotropic virus 1; HTLV-I antibodies; lymphoproliferative disorders; sentinel surveillance; seroepidemiologic studies; Cuba. 